\title{
Comparative Effectiveness of Fecal Immunochemical Tests versus Flexible Sigmoidoscopy for Colorectal Cancer Screening: A Systematic Review and Meta-Analysis of Randomized Clinical Trials
}

\author{
Hemant Raj Mutneja ${ }^{1}$, Rohit Agrawal ${ }^{2}$, Abhishek Bhurwal ${ }^{3}$, Shilpa Arora ${ }^{4}$, Andrew Go ${ }^{5}$, Bashar M Attar ${ }^{1,4}$
}

1) John H Stroger, Jr. Hospital

of Cook County, Chicago,

Illinois;

2) University of Illinois,

Chicago, Illinois;

3) Robert Wood Johnson

University Hospital, New

Brunswick, New Jersey;

4) Rush University Medical

Center, Chicago, Illinois;

5) Loyola University Medical

Center, Chicago,

Illinois, USA

\author{
Address for correspondence: \\ Hemant Raj Mutneja MD \\ 1950 W Polk St, $6^{\text {th }}$ Floor, \\ Chicago, Illinois, 60612, \\ United States of America \\ hemantmutneja@gmail.com, \\ hmutneja@cookcountyhhs.org
}

Received: 30.01 .2021 Accepted: 12.02.2021

\begin{abstract}
Background \& Aims: Fecal immunochemical tests (FITs) and flexible sigmoidoscopies are commonly used modalities for colorectal cancer (CRC) screening. We performed a systematic review and meta-analysis to compare the effectiveness of FIT and sigmoidoscopy in CRC screening.

Methods: PRISMA statement and Cochrane guidelines were followed for this review. Digital dissertation databases were searched from inception till December 1st 2020 and randomized clinical trials comparing the detection rates of CRC for FIT and sigmoidoscopy were included. Outcomes for analysis included participation rates and detection rates of CRC, advanced adenomas and advanced colorectal neoplasia for both screening modalities.

Results: Five randomized clinical trials with a total of 261,755 patients were included for the analysis. The participation rate for FIT was significantly higher compared to flexible sigmoidoscopy (OR 2.11, 95\% CI 1.29-3.44, $\mathrm{p}=0.003$ ). In intention-to-screen analysis, the detection rate for advanced colorectal neoplasia was significantly lower with FIT (OR 0.62, 95\% CI 0.45-0.84, p=0.002) as compared to flexible sigmoidoscopy but not statistically different for CRC (OR 1.15, 95\% CI 0.65-2.02, $\mathrm{p}=0.63$ ).

Conclusion: Despite lower participation amongst patients, CRC screening with flexible sigmoidoscopy leads to higher detection of advanced colorectal neoplasia, when compared to a single round of fecal immunochemical testing.
\end{abstract}

Key words: colorectal cancer screening - sigmoidoscopy - fecal immunochemical test - meta-analysis.

Abbreviations: CRC: colorectal cancer; FIT: fecal immunochemical test; gFOBT: guaiac-based fecal occult blood test; OR: odds ratio.

\section{INTRODUCTION}

Colorectal cancer (CRC) is the fourth most commonly diagnosed cancer in the world [1]. It accounted for nearly 1.8 million new cases worldwide in 2018 [2]. However, the incidence and mortality rates of CRC seem to be decreasing in the developed world [3]. This has largely been attributed to a combination of better screening modalities and more effective treatment. Several stool and visualization-based tools, such as the guaiac-based fecal occult blood test (gFOBT), fecal immunochemical test (FIT), FIT-DNA, colonoscopy, flexible sigmoidoscopy and computerized tomography colonography have been shown to reduce the incidence rate for CRC [4]. Colonoscopy, the most utilized screening test, with the highest sensitivity and specificity, has the added advantage of being able to perform therapeutics $[5,6]$. Flexible sigmoidoscopy has been shown to have similar efficacy to colonoscopy in identifying left-sided lesions with a reduction in incidence and mortality rates by $25 \%$ and $30 \%$ respectively, when compared to no screening or usual care [7-11]. Fecal immunochemical test has been reported to have better sensitivity than gFOBT in detecting CRCs and advanced adenomas and has largely replaced gFOBT as the fecal test of choice [12-16].

Despite the well-known efficacy of these tools, the full benefit is undermined by their underutilization and low adherence rates. Some factors associated with underutilization of endoscopic screening are cumbersome bowel preparations, need for sedation or anesthesia, invasiveness of the procedures and potential complications [17]. A meta-analysis in 2012 suggested that higher detection rates associated with 
endoscopy minimized any impact of lower adherence rates in a screening setting [13]. However, the analysis was limited by a large number of studies utilizing gFOBT which has largely been replaced by FIT now. Several studies have since then evaluated FIT and compared it to flexible sigmoidoscopy as a screening technique for CRC [18-22]. We hereby present a systematic review and meta-analysis of randomized trials comparing detection and participation rates of FIT and flexible sigmoidoscopy in CRC screening.

\section{METHODS}

Our study was performed in accordance with the Preferred Reporting Items for Systematic reviews and Meta-Analyses (PRISMA) statement and Cochrane guidelines for systematic reviews $[23,24]$.

\section{Search Strategy}

The search strategy was designed and conducted by the authors. Three reviewers (H.R.M., R.A. \& A.B.) independently and in duplicate searched PubMed, Cochrane Central Register of Controlled Trials (CENTRAL), CINAHL, Scopus and Google Scholar. using multiple search terms ('fecal immunochemical test,' 'FIT', 'FOBT', 'sigmoidoscopy', 'endoscopy') from inception to December $1^{\text {st }} 2020$ (Supplementary file). All titles and abstracts were identified by the authors and screened to accrue potentially eligible studies. Then, the same reviewers independently assessed all selected full-text manuscripts for the eligibility. Disagreements between two reviewers were resolved through consensus and after input from the third reviewer and principal investigator.

\section{Eligibility Criteria}

The specific inclusion criteria for the systematic review and meta-analysis were: (1) all randomized trials in patients more than 50 years of age comparing FITs to flexible sigmoidoscopies as strategies for CRC screening; (2) studies with information available to evaluate the detection rates of CRC based on screening strategy used; (3) full text articles available in English language. Thus, reviewed studies included in our analysis were randomized trials comparing the detection rates of CRC with FITs to flexible sigmoidoscopies. Non-randomized studies and studies evaluating gFOBT were excluded from the analysis.

\section{Study Characteristics and Quality assessment}

We selected data collection forms for randomized trials based on Cochrane Collaboration risk assessment tool to adhere to principles of sound methodological quality [25]. For each study, we ascertained seven domains to identify imbalances in baseline characteristics. We used the terms "low risk" and "high risk" of bias at the study level for scoring system. In our study, "unclear bias" was judged from baseline imbalance which could not be ascertained from the seven domains.

Quality assessments were also conducted independently, and discrepancies were resolved by consensus.

\section{Outcome measures}

The outcomes for this systematic review and meta-analysis were: 1) participation rates amongst patients offered screening with FITs and flexible sigmoidoscopies, and 2) detection rates of CRC, advanced adenomas and advanced colorectal neoplasia (CRC and advanced adenoma) amongst patients subjected to screening with FITs and sigmoidoscopies. A per-protocol analysis (per-screenee) was performed to calculate detection rate in the population that underwent the screening test and an intention-to-screen analysis (per-invitee) was performed to calculate the detection rate in the population that was offered screening, irrespective of participation.

\section{Data Extraction}

Three reviewers (H.R.M., R.A., A.B.) independently reviewed and abstracted data on detection and participation rates for each eligible study. If there were multiple reports stemming from a specific study database, data from the most robust study was extracted with other studies contributing towards bibliography. The reviewers sorted the data separately in all stages of study collection, data extraction and quality assessment. All discrepancies found between 2 reviewers were resolved with consensus and inputs from other authors.

\section{Quantitative data synthesis}

All data were analyzed using the computer software (Review Manager (RevMan), version 5.4.1, the Cochrane Collaboration, 2020). The final pooled risk estimates were obtained using random effects models by the methods of DerSimonian and Laird with inverse variance weighting. Raw data for detection and participation events and nonevents from each study were used to calculate a crude odds ratio (OR) for each study. The Cochrane $\mathrm{Q}$ and the $\mathrm{I}^{2}$ statistics were calculated to assess heterogeneity between studies. $\mathrm{P}<0.10$ for chi-square test and $\mathrm{I}^{2}<20 \%$ were interpreted as low-level heterogeneity.

\section{RESULTS}

\section{Results of the Search}

The initial library search identified 1,435 potentially relevant citations from PubMed, Medline, CENTRAL, EMBASE, Scopus and clinical trial registries. Subsequently, after removal of duplicates, 1,014 underwent title and abstract review. The remaining manuscripts were scrutinized further and finally, five studies were included in the full review. There was no overlap of patients among the different studies. The PRISMA flowchart for the search strategy is shown in Fig. 1.

\section{Included Studies}

Five studies with a total of 261,755 patients were included in the review. All the studies were randomized, population-based clinical trials. The cut-offs for FIT positivity varied across the studies: $100 \mu \mathrm{g} / \mathrm{g}$ for Segnan et al. [18], $20 \mu \mathrm{g} / \mathrm{g}$ for Hol et al. [19], $15 \mu \mathrm{g} / \mathrm{g}$ for Castells et al. [20] \& Randel et al. [22] and 10 $\mu \mathrm{g} / \mathrm{g}$ for Grobbee et al. [21]. The characteristics of the included studies are shown in Table I.

\section{Risk of Bias in Included Studies}

All the included studies reported adequate methods of randomization except for Grobbee et al. [21] where details of the randomization process were not described. None of the included studies reported adequate concealment to prevent 


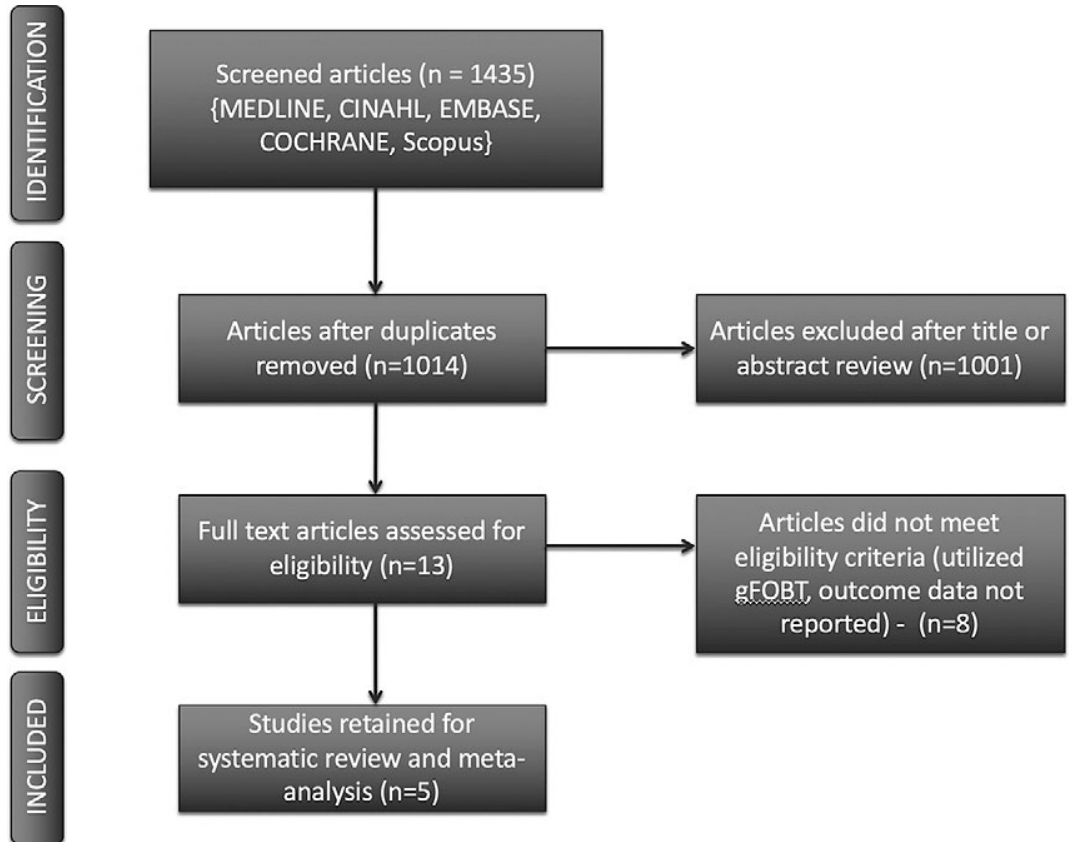

Fig. 1. PRISMA flowchart for review process.

selection bias or detection bias. Blinding of participants and personnel was not possible, which is usually the scenario in endoscopy studies, and therefore could lead to performance bias. All the studies had low risk of attrition bias except for Randel et al [22]. Castells et al [20] extrapolated sigmoidoscopy results from colonoscopy. Grobbee et al. [21] and Hol et al. [19] have described possible selection bias in the results. Therefore, overall, the studies are fair in quality with regards to the risk of bias. The quality assessment has been illustrated in Fig. 2 .

Due to the low number of included studies $(n<10)$, our meta-analysis is underpowered to detect any publication bias.

\section{Outcome Analysis}

The odds of participation were higher with FIT as compared to flexible sigmoidoscopy (OR: 2.11, 95\%CI: 1.29-3.44, $\mathrm{p}=0.003$ ). The Forest Plot for this analysis has been shown in Fig. 3.

In per-protocol analysis, the odds of detection of CRC were lower with FIT as compared to flexible sigmoidoscopy
(OR: 0.76, 95\%CI: 0.61-0.96, $\mathrm{p}=0.02$ ). This analysis has been shown in Fig. 4.

In intention-to-screen analysis, there was no statistically significant difference in the detection rate of CRC amongst the two groups (OR: 1.15, 95\%CI: 0.65-2.02, $\mathrm{p}=0.63$ ). This has been shown in Fig. 5 .

In per-protocol analysis, the odds of detection of advanced colorectal neoplasia were significantly lower in FIT group compared to flexible sigmoidoscopy group (OR: 0.40, 95\%CI: 0.32-0.48, $\mathrm{p}<0.001)$. This has been shown in Fig. 6 .

In intention-to-screen analysis, FIT was associated with a lower detection rate of advanced neoplasia as compared to flexible sigmoidoscopy (OR: 0.62, 95\%CI: 0.45-0.84, $\mathrm{p}=0.002$ ). This has been illustrated in Fig. 7. The odds of detection of advanced adenoma were significantly lower in FIT group compared to flexible sigmoidoscopy in both per-protocol (OR: 0.37, 95\% CI: 0.30-0.46, p<0.001) and intention-to-screen (OR: 0.58, 95\%CI: $0.43-0.79, \mathrm{p}<0.001)$ analysis. The Forest Plots for these analyses are shown in Figs. 8 and 9 respectively.

Table I. Characteristics of included studies

\begin{tabular}{|c|c|c|c|c|c|c|c|}
\hline Study & Year & Design & Country & $\begin{array}{l}\text { Patient Age } \\
\text { (years) }\end{array}$ & $\begin{array}{l}\text { Total number } \\
\text { of patients }\end{array}$ & Sigmoidoscopy & $\begin{array}{l}\text { FIT type and cut-off for } \\
\text { positivity (Hb/feces) }\end{array}$ \\
\hline $\begin{array}{l}\text { Segnan et al. } \\
{[18]}\end{array}$ & 2007 & $\begin{array}{l}\text { Population-based } \\
\text { Multicenter Randomized } \\
\text { Trial }\end{array}$ & Italy & $55-64$ & 20,042 & $\begin{array}{l}\text { Flexible } \\
\text { Sigmoidoscopy }\end{array}$ & Immudia HemSp, $100 \mu \mathrm{g} / \mathrm{g}$ \\
\hline Hol et al. [19] & 2010 & $\begin{array}{l}\text { Population-based } \\
\text { Randomized Trial }\end{array}$ & Netherlands & $50-74$ & 15,011 & $\begin{array}{l}\text { Flexible } \\
\text { Sigmoidoscopy }\end{array}$ & OC sensor, $20 \mu \mathrm{g} / \mathrm{g}$ \\
\hline $\begin{array}{l}\text { Castells et al. } \\
{[20]}\end{array}$ & 2014 & $\begin{array}{l}\text { Population-based } \\
\text { Multicenter Randomized } \\
\text { Trial }\end{array}$ & Spain & $50-69$ & 57,404 & $\begin{array}{l}\text { Estimated by } \\
\text { Colonoscopy }\end{array}$ & OC sensor, $15 \mu \mathrm{g} / \mathrm{g}$ \\
\hline $\begin{array}{l}\text { Grobbee et al. } \\
{[21]}\end{array}$ & 2020 & $\begin{array}{l}\text { Population-based } \\
\text { Randomized Trial }\end{array}$ & Netherlands & $50-74$ & 30,007 & $\begin{array}{l}\text { Flexible } \\
\text { Sigmoidoscopy }\end{array}$ & Not specified, $10 \mu \mathrm{g} / \mathrm{g}$ \\
\hline Randel et al22 & 2020 & $\begin{array}{l}\text { Population-based } \\
\text { Randomized Trial }\end{array}$ & Norway & $50-74$ & 139,291 & $\begin{array}{l}\text { Flexible } \\
\text { Sigmoidoscopy }\end{array}$ & OC Sensor-Diana, $15 \mu \mathrm{g} / \mathrm{g}$ \\
\hline
\end{tabular}

FIT: fecal immunochemical test. 


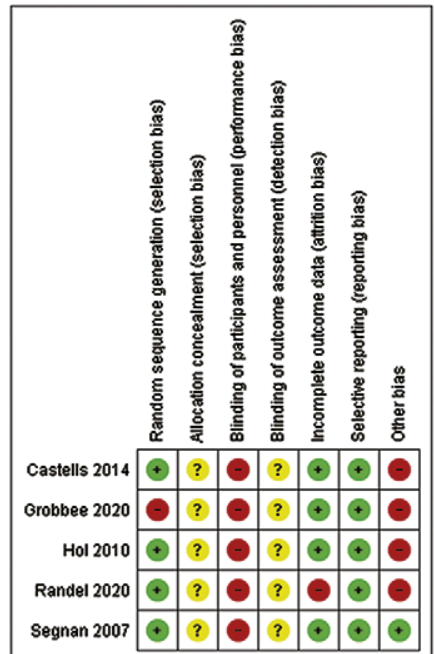

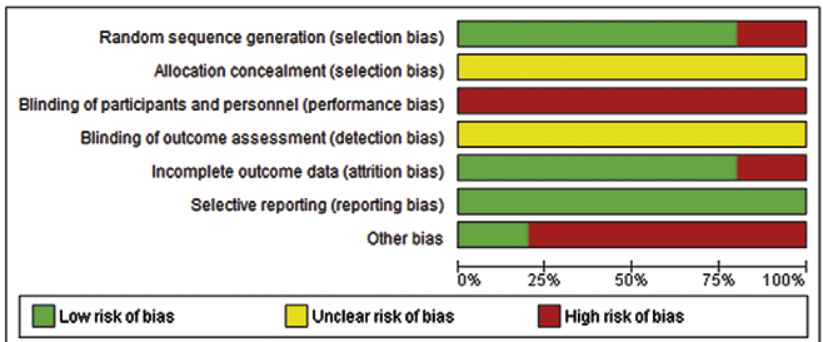

Risk of bias graph: review authors' judgements about each risk of bias item presented as percentages across all included studies.

Risk of bias summary: review authors' judgements about each risk of bias item for each included study.

Fig. 2. Quality Assessment of Included Studies.

\begin{tabular}{|c|c|c|c|c|c|c|c|c|c|c|c|}
\hline \multirow[b]{2}{*}{ Study or Subgroup } & \multicolumn{2}{|c|}{ FIT } & \multicolumn{2}{|c|}{ Sigmoidoscopy } & \multicolumn{3}{|c|}{ Odds Ratio } & \multirow{2}{*}{\multicolumn{4}{|c|}{$\begin{array}{l}\text { Odds Ratio } \\
\text { M-H, Random, } 95 \% \mathrm{Cl}\end{array}$}} \\
\hline & Events & Total & Events & Total & Weight & $\mathrm{M}-\mathrm{H}$, Random, $95 \% \mathrm{Cl}$ & Year & & & & \\
\hline Segnan 2007 & 1965 & 6075 & 1944 & 6018 & $20.0 \%$ & $1.00[0.93,1.08]$ & 2007 & & 7 & 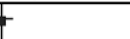 & \\
\hline $\mathrm{Hol} 2010$ & 2979 & 4843 & 1522 & 4700 & $19.9 \%$ & $3.34[3.07,3.63]$ & 2010 & & & & $\rightarrow$ \\
\hline Castells 2014 & 10611 & 26599 & 5059 & 26703 & $20.0 \%$ & $2.84[2.73,2.95]$ & 2014 & & & & . \\
\hline Grobbee 2020 & 8847 & 14651 & 2435 & 7882 & $20.0 \%$ & $3.41[3.22,3.61]$ & 2020 & & & & $=$ \\
\hline Randel 2020 & 40966 & 70096 & 36065 & 69195 & $20.1 \%$ & $1.29[1.26,1.32]$ & 2020 & & & - & \\
\hline Total $(95 \% \mathrm{Cl})$ & & 122264 & & 114498 & $100.0 \%$ & $2.11[1.29,3.44]$ & & & & & \\
\hline Total events & 65368 & & 47025 & & & & & & & & \\
\hline $\begin{array}{l}\text { Heterogeneity: } \mathrm{Tau}^{2}= \\
\text { Test for overall effect }\end{array}$ & $\begin{array}{l}0.31 ; C h \\
Z=2.99\end{array}$ & $\begin{array}{l}\mathrm{i}^{2}=2280 \\
(\mathrm{P}=0.0\end{array}$ & $\begin{array}{l}0.43, \mathrm{df}= \\
03)\end{array}$ & $4(P<0 . C$ & 00001); & ${ }^{2}=100 \%$ & & 0.2 & $\begin{array}{ll}0.5 & 1 \\
\text { moidoscopy }\end{array}$ & $\begin{array}{l}2 \\
2 \\
\text { Favors FIT }\end{array}$ & \\
\hline
\end{tabular}

Fig. 3. Forest Plot for comparison of participation rate.

FIT Sigmoidoscopy Odds Ratio

Study or Subgroup Events Total Events Total Weight M-H, Random, 95\% Cl Year

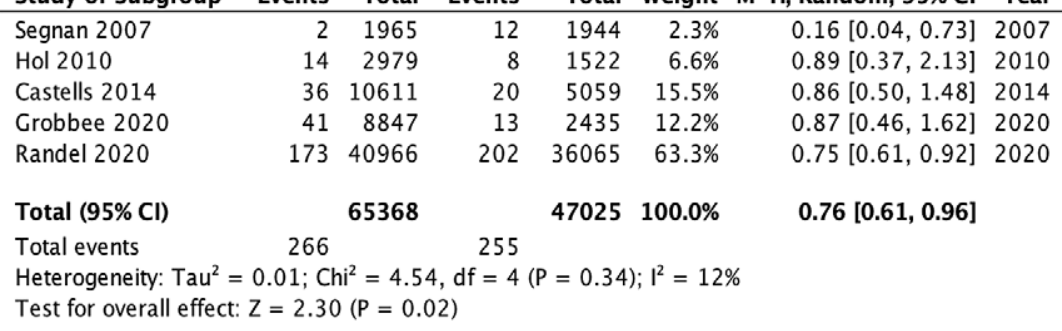

Fig. 4. Forest Plot for comparison of detection rate of colorectal cancer in per-protocol analysis.
Odds Ratio

M-H, Random, 95\% Cl

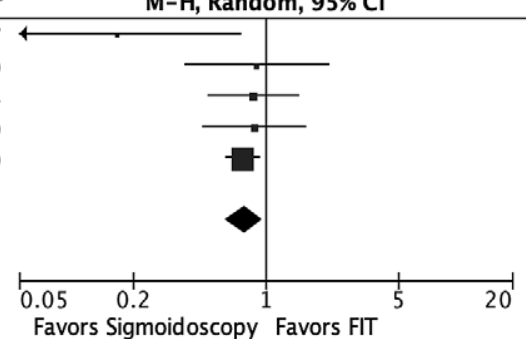

\begin{tabular}{|c|c|c|c|c|c|c|c|c|c|c|}
\hline \multirow[b]{2}{*}{ Study or Subgroup } & \multicolumn{2}{|c|}{ FIT } & \multicolumn{2}{|c|}{ Sigmoidoscopy } & \multicolumn{3}{|c|}{ Odds Ratio } & \multirow{2}{*}{\multicolumn{2}{|c|}{$\begin{array}{c}\text { Odds Ratio } \\
\text { M-H, Random, } 95 \% \mathrm{Cl}\end{array}$}} & \\
\hline & Events & Total & Events & Total & Weight & M-H, Random, $95 \% \mathrm{Cl}$ & Year & & & \\
\hline Segnan 2007 & 2 & 6075 & 12 & 6018 & $9.6 \%$ & $0.16[0.04,0.74]$ & 2007 & $\leftarrow$ & & \\
\hline $\mathrm{Hol} 2010$ & 14 & 4843 & 8 & 4700 & $17.4 \%$ & $1.70[0.71,4.06]$ & 2010 & & & \\
\hline Castells 2014 & 36 & 26599 & 20 & 26703 & $23.2 \%$ & $1.81[1.05,3.12]$ & 2014 & & & \\
\hline Grobbee 2020 & 41 & 14651 & 12 & 7882 & $21.4 \%$ & $1.84[0.97,3.50]$ & 2020 & & & \\
\hline Randel 2020 & 173 & 70096 & 202 & 69195 & $28.5 \%$ & $0.85[0.69,1.04]$ & 2020 & & & \\
\hline Total $(95 \% \mathrm{CI})$ & & 122264 & & 114498 & $100.0 \%$ & $1.15[0.65,2.02]$ & & & & \\
\hline Total events & 266 & & 254 & & & & & & & \\
\hline $\begin{array}{l}\text { Heterogeneity: } \mathrm{Tau}^{2}= \\
\text { Test for overall effect }\end{array}$ & $\begin{array}{l}0.28 ; C h i \\
Z=0.48\end{array}$ & $\begin{array}{l}\mathrm{i}^{2}=17.53 \\
(\mathrm{P}=0.63\end{array}$ & 3, $d f=4$ & $P=0.002$ & 2); $I^{2}=77$ & & & $\begin{array}{lll}0.05 & 0.2 \\
\text { Favors } & \text { Sigmoidoscopy }\end{array}$ & Favors FIT & 20 \\
\hline
\end{tabular}

Fig. 5. Forest Plot for comparison of detection rate of colorectal cancer in intention-to-screen analysis. 


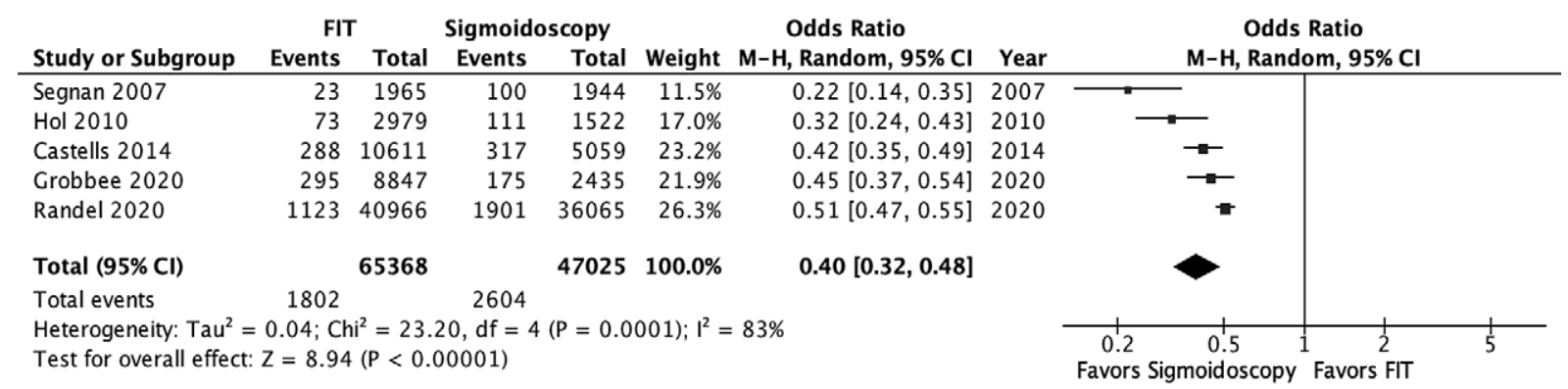

Fig. 6. Forest Plot for comparison of detection rate of advanced colorectal neoplasia in per-protocol analysis.

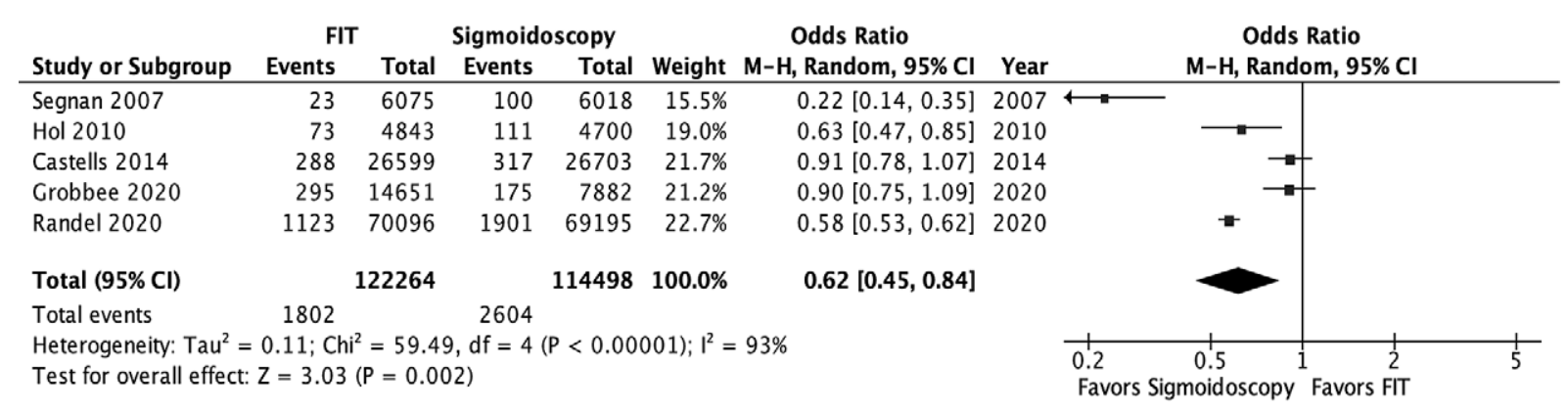

Fig. 7. Forest Plot for comparison of detection rate of advanced colorectal neoplasia in intention-to-screen analysis.

\begin{tabular}{|c|c|c|c|c|c|c|c|c|c|}
\hline \multirow{2}{*}{$\begin{array}{l}\text { Study or Subgroup } \\
\text { Segnan } 2007\end{array}$} & $\begin{array}{r}\text { FIT } \\
\text { Events }\end{array}$ & Total & $\begin{array}{l}\text { Sigmoid } \\
\text { Events }\end{array}$ & $\begin{array}{l}\text { scopy } \\
\text { Total }\end{array}$ & Weight & \multirow{2}{*}{$\begin{array}{c}\text { Odds Ratio } \\
\text { M-H, Random, 95\% Cl } \\
0.23[0.14,0.37]\end{array}$} & \multicolumn{3}{|c|}{$\begin{array}{c}\text { Odds Ratio } \\
\mathrm{M}-\mathrm{H} \text {, Random, } 95 \% \mathrm{Cl}\end{array}$} \\
\hline & 21 & 1965 & 88 & 1944 & $11.2 \%$ & & 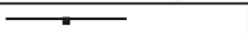 & & \\
\hline Hol 2010 & 59 & 2979 & 103 & 1522 & $16.5 \%$ & $0.28[0.20,0.39]$ & 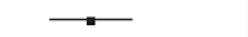 & & \\
\hline Castells 2014 & 252 & 10611 & 297 & 5059 & $23.4 \%$ & $0.39[0.33,0.46]$ & $\rightarrow$ & & \\
\hline Grobbee 2020 & 254 & 8847 & 162 & 2435 & $22.1 \%$ & $0.41[0.34,0.51]$ & $\rightarrow-$ & & \\
\hline Randel 2020 & 950 & 40966 & 1699 & 36065 & $26.8 \%$ & $0.48[0.44,0.52]$ & $=$ & & \\
\hline Total $(95 \% \mathrm{Cl})$ & & 65368 & & 47025 & $100.0 \%$ & $0.37[0.30,0.46]$ & & & \\
\hline Total events & 1536 & & 2349 & & & & & & \\
\hline $\begin{array}{l}\text { Heterogeneity: } \mathrm{Tau}^{2}= \\
\text { Test for overall effect: }\end{array}$ & $\begin{array}{l}0.04 ; \mathrm{Ch} \\
\mathrm{Z}=9.32\end{array}$ & $\begin{array}{l}2=21 \\
(P<0\end{array}$ & $\begin{array}{l}1, \mathrm{df}=4 \\
0001)\end{array}$ & $P=0$ & 03); & $\%$ & $\begin{array}{cc}1 & 0.5 \\
\text { Favors } & \text { Sigmoidoscopy }\end{array}$ & 1 Favors FIT & 5 \\
\hline
\end{tabular}

Fig. 8. Forest Plot for comparison of detection rate of advanced adenoma in per-protocol analysis.

\begin{tabular}{|c|c|c|c|c|c|c|c|c|c|c|c|}
\hline \multirow{3}{*}{$\begin{array}{l}\text { Study or Subgroup } \\
\text { Segnan } 2007\end{array}$} & \multicolumn{2}{|c|}{ FIT } & \multicolumn{2}{|c|}{ Sigmoidoscopy } & \multicolumn{3}{|c|}{ Odds Ratio } & \multirow{2}{*}{\multicolumn{4}{|c|}{$\begin{array}{c}\text { Odds Ratio } \\
\text { M-H, Random, } 95 \% \mathrm{Cl}\end{array}$}} \\
\hline & Events & Total & Events & Total & Weight & M-H, Random, 95\% Cl & Year & & & & \\
\hline & 21 & 6075 & 88 & 6018 & $14.7 \%$ & $0.23[0.15,0.38]$ & 2007 & $\longrightarrow$ & & & \\
\hline Hol 2010 & 59 & 4843 & 103 & 4700 & $18.5 \%$ & $0.55[0.40,0.76]$ & 2010 & & & & \\
\hline Castells 2014 & 252 & 26599 & 297 & 26703 & $22.0 \%$ & $0.85[0.72,1.01]$ & 2014 & $\rightarrow-$ & & & \\
\hline Grobbee 2020 & 254 & 14651 & 163 & 7882 & $21.4 \%$ & $0.84[0.68,1.02]$ & 2020 & $\rightarrow-$ & & & \\
\hline Randel 2020 & 950 & 70096 & 1699 & 69195 & $23.3 \%$ & $0.55[0.50,0.59]$ & 2020 & 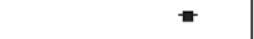 & & & \\
\hline Total $(95 \% \mathrm{Cl})$ & & 122264 & & 114498 & $100.0 \%$ & $0.58[0.43,0.79]$ & & & & & \\
\hline Total events & 1536 & & 2350 & & & & & & & & \\
\hline $\begin{array}{l}\text { Heterogeneity: } \mathrm{Tau}^{2} \\
\text { Test for overall effec }\end{array}$ & $\begin{array}{l}0.10 ; C h \\
Z=3.55\end{array}$ & $\begin{array}{l}2=47.7 \\
(P=0.0\end{array}$ & $\begin{array}{l}5, \mathrm{df}=4( \\
004)\end{array}$ & $(P<0 . C$ & $001) ; 1^{2}$ & $=92 \%$ & & \begin{tabular}{|ccc|} 
& $\mid$ & $\mid$ \\
0.1 & 0.2 & 0.5 \\
\multicolumn{3}{l}{ Favors Sigmoidoscopy }
\end{tabular} & $\begin{array}{l}1 \\
1 \\
\text { Favors FIT }\end{array}$ & 5 & \\
\hline
\end{tabular}

Fig. 9. Forest Plot for comparison of detection rate of advanced adenoma in intention-to-screen analysis.

\section{DISCUSSION}

In this systematic review and meta-analysis of five randomized trials with a total of 261,755 patients, we found that flexible sigmoidoscopies are associated with higher CRC and advanced neoplasia detection rates but lower participation rates, as compared to FITs. The overall diagnostic yield of advanced colorectal neoplasia for flexible sigmoidoscopies is significantly higher as compared to FITs but that of CRC is not statistically different between the two screening modalities.
A previous meta-analysis comparing different colorectal screening techniques also found a higher diagnostic yield of colorectal neoplasia with endoscopic screening when compared with fecal tests [13]. However, that data was based predominantly on gFOBT which have been found to be inferior to FITs $[15,16]$. Also, in their analysis, endoscopic screening comprised of both flexible sigmoidoscopy and colonoscopy, such that a direct comparison of sigmoidoscopy with FIT was not possible.

The detection of advanced neoplasia is a relevant intermediate outcome of colorectal screening studies [18]. In 
our intention-to-screen analysis, FIT has a significantly lower detection rate of advanced colorectal neoplasia as compared to flexible sigmoidoscopy. This is largely driven by a lower detection rate of advanced adenomas as the detection rates of CRC were not statistically different between the two groups. As advanced adenomas are considered a surrogate marker of CRC [26], their higher detection with sigmoidoscopy confers an advantage in reducing the incidence of CRC over a screening interval in a population [27].

Another important outcome in colorectal screening is the participation rate for different screening modalities across the population. Our analysis confirms lower odds of participation with sigmoidoscopy compared to fecal testing, as has been the case previously [28]. This is likely attributable to invasiveness of the procedure, risk of potential complications and the need for complex infrastructure associated with it. The participation rates differ across the included studies highlighting the fact that other factors like patient awareness and effectiveness of screening program influence patient participation at a community level [29].

It is to be noted that our results are a comparison of the diagnostic yields of 'once only' flexible sigmoidoscopy with a single round of FIT. In a screening programme, multiple rounds of fecal testing would have been undertaken in a 5 or 10-year period over which a single flexible sigmoidoscopy is usually performed. Therefore, the true diagnostic yield of FIT in a given screening interval would be expected to be higher due to multiple rounds being administered and higher participation at the community level. Unfortunately, three of the included studies only report data over the first round of FIT and therefore, comparison of detection rates over a longer time period was not possible in our analysis.

A major limiting factor affecting CRC screening in a population is the endoscopic capacity of that region [30]. A potential benefit with stool testing is that it helps identify higher-risk individuals who can be selectively referred for colonoscopy. It is, however, to be noted that the FIT-positive patients need timely follow up colonoscopies as delaying colonoscopies in these patients has been shown to be associated with higher incidence of CRC [31]. Careful planning and implementation is therefore needed to devise efficient colorectal cancer screening programmes.

There are certain limitations to our analysis. As stated previously, the results may skew towards FIT when multiple rounds of FIT are compared to single flexible sigmoidoscopy over a given screening interval. There is heterogeneity amongst the studies, especially in terms of the participation rates. This is likely accounted by the cultural and organizational differences amongst the study populations and perhaps, by the large size of the populations as well. However, given the large difference in our outcome analysis, this heterogeneity is unlikely to be of any clinical significance. Also, blinding is not feasible in endoscopy-based studies and is an inherent limitation that could lead to a performance bias. However, the included studies have uniformity in outcome definition and therefore, the heterogeneity is low. Also, the cut-offs for FIT positivity varied ten-fold across the studies. The Segnan study [18] used a cut-off of $100 \mu \mathrm{g} / \mathrm{g} \mathrm{Hb} /$ feces for FIT positivity. This is one of the earlier studies using FITs when they were newly implemented in population-based studies. The cut-offs for positivity have since then been revised and are much lower now. The expected influence of this variability between studies was hard to address specifically even by using random-effects models for conducting the meta-analysis. Lastly, our metaanalysis is underpowered to detect any publication bias due to the small number of included studies.

\section{CONCLUSIONS}

Despite lower participation amongst patients, CRC screening with flexible sigmoidoscopy leads to higher detection of advanced colorectal neoplasia, when compared to a single round of FIT. Future data on mortality reduction is needed to differentiate between the two screening techniques.

Conflicts of interest: None to declare.

Authors' contributions: H.M., A.B. conceived and designed the study, performed the statistical analysis. H.M., A.B., R.A. searched the literature, collected the data, interpreted the results. H.M., R.A., S.A., A.G. drafted the paper. H.M., B.A., A.G., S.A. revised the manuscript for important intellectual content. All the authors approved the final version of the manuscript.

Supplementary material: To access the supplementary material visit the online version of the J Gastrointestin Liver Dis at http://dx.doi. org/10.15403/jgld-3432

\section{REFERENCES}

1. Rawla P, Sunkara T, Barsouk A. Epidemiology of colorectal cancer: incidence, mortality, survival, and risk factors. Prz Gastroenterol 2019;14:89-103. doi:10.5114/pg.2018.81072

2. Bray F, Ferlay J, Soerjomataram I, Siegel RL, Torre LA, Jemal A. Global cancer statistics 2018: GLOBOCAN estimates of incidence and mortality worldwide for 36 cancers in 185 countries. CA Cancer J Clin 2018;68:394-424. doi:10.3322/caac. 21492

3. Arnold M, Sierra MS, Laversanne M, Soerjomataram I, Jemal A, Bray F. Global patterns and trends in colorectal cancer incidence and mortality. Gut 2017;66:683-691. doi:10.1136/gutjnl-2015-310912

4. US Preventive Services Task Force; Bibbins-Domingo K, Grossman DC, Curry SJ, et al. Screening for Colorectal Cancer: US Preventive Services Task Force Recommendation Statement. JAMA 2016;315:2564-2575. doi:10.1001/jama.2016.5989

5. Zauber A, Knudsen A, Rutter CM, Lansdorp-Vogelaar I, Kuntz. Evaluating the Benefits and Harms of Colorectal Cancer Screening Strategies: A Collaborative Modeling Approach. AHRQ Publication No. 14-05203-EF-2. Rockville, MD: Agency for Healthcare Research and Quality; October 2015.

6. Knudsen AB, Zauber AG, Rutter CM, et al. Estimation of Benefits, Burden, and Harms of Colorectal Cancer Screening Strategies: Modeling Study for the US Preventive Services Task Force. JAMA 2016;315:25952609. doi:10.1001/jama.2016.6828

7. Nishihara R, Wu K, Lochhead P, et al. Long-term colorectal-cancer incidence and mortality after lower endoscopy. N Engl J Med 2013;369:1095-1105. doi:10.1056/NEJMoa1301969 
8. Atkin W, Wooldrage K, Parkin DM, et al. Long term effects of once-only flexible sigmoidoscopy screening after 17 years of follow-up: the UK Flexible Sigmoidoscopy Screening randomised controlled trial. Lancet 2017;389:1299-1311. doi:10.1016/S0140-6736(17)30396-3

9. Segnan N, Armaroli P, Bonelli L, et al; SCORE Working Group. Onceonly sigmoidoscopy in colorectal cancer screening: follow-up findings of the Italian Randomized Controlled Trial--SCORE. J Natl Cancer Inst 2011;103:1310-1322. doi:10.1093/jnci/djr284

10. Holme $\varnothing$, Løberg $M$, Kalager $M$, et al. Effect of flexible sigmoidoscopy screening on colorectal cancer incidence and mortality: a randomized clinical trial. JAMA 2014;312:606-615. doi:10.1001/jama.2014.8266

11. Miller EA, Pinsky PF, Schoen RE, Prorok PC, Church TR. Effect of flexible sigmoidoscopy screening on colorectal cancer incidence and mortality: long-term follow-up of the randomised US PLCO cancer screening trial. Lancet Gastroenterol Hepatol 2019;4:101-110. doi:10.1016/S2468-1253(18)30358-3

12. Halloran SP, Launoy G, Zappa M; International Agency for Research on Cancer. European guidelines for quality assurance in colorectal cancer screening and diagnosis. First Edition--Faecal occult blood testing. Endoscopy 2012;44 Suppl 3:SE65-SE87. doi:10.1055/s-0032-1309791

13. Hassan C, Giorgi Rossi P, Camilloni L, et al; HTA Group. Meta-analysis: adherence to colorectal cancer screening and the detection rate for advanced neoplasia, according to the type of screening test. Aliment Pharmacol Ther 2012;36:929-940. doi:10.1111/apt.12071

14. Robertson DJ, Lee JK, Boland CR, et al. Recommendations on Fecal Immunochemical Testing to Screen for Colorectal Neoplasia: A Consensus Statement by the US Multi-Society Task Force on Colorectal Cancer. Gastroenterology 2017;152:1217-1237.e3. doi:10.1053/j. gastro.2016.08.053

15. Meklin J, Syrjänen K, Eskelinen M. Colorectal Cancer Screening With Traditional and New-generation Fecal Immunochemical Tests: A Critical Review of Fecal Occult Blood Tests. Anticancer Res 2020;40:575-581. doi:10.21873/anticanres.13987

16. Meklin J, Syrjänen K, Eskelinen M. Fecal Occult Blood Tests in Colorectal Cancer Screening: Systematic Review and Meta-analysis of Traditional and New-generation Fecal Immunochemical Tests. Anticancer Res 2020;40:3591-3604. doi:10.21873/anticanres.14349

17. Finney Rutten LJ, Jacobson DJ, Jenkins GD, et al. Colorectal cancer screening completion: An examination of differences by screening modality. Prev Med Rep 2020;20:101202. doi:10.1016/j. pmedr.2020.101202

18. Segnan N, Senore C, Andreoni B, et al; SCORE3 Working GroupItaly. Comparing attendance and detection rate of colonoscopy with sigmoidoscopy and FIT for colorectal cancer screening. Gastroenterology 2007;132:2304-2312. doi:10.1053/j.gastro.2007.03.030

19. Hol L, van Leerdam ME, van Ballegooijen M, et al. Screening for colorectal cancer: randomised trial comparing guaiac-based and immunochemical faecal occult blood testing and flexible sigmoidoscopy. Gut 2010;59:62-68. doi:10.1136/gut.2009.177089

20 Castells A, Quintero E, Álvarez C, et al; COLONPREV Study Investigators. Rate of detection of advanced neoplasms in proximal colon by simulated sigmoidoscopy vs fecal immunochemical tests. Clin Gastroenterol Hepatol 2014;12:1708-1716.e4. doi:10.1016/j.cgh.2014.03.022

21. Grobbee EJ, van der Vlugt M, van Vuuren AJ, et al. Diagnostic Yield of One-Time Colonoscopy vs One-Time Flexible Sigmoidoscopy vs Multiple Rounds of Mailed Fecal Immunohistochemical Tests in Colorectal Cancer Screening. Clin Gastroenterol Hepatol 2020;18:667675.e1. doi:10.1016/j.cgh.2019.08.015

22. Randel KR, Schult AL, Botteri E, et al. Colorectal cancer screening with repeated fecal immunochemical test versus sigmoidoscopy: baseline results from a randomized trial. Gastroenterology 2021;160:1085-1096. e5. doi:10.1053/j.gastro.2020.11.037

23. Moher D, Liberati A, Tetzlaff J, Altman DG; PRISMA Group. Preferred reporting items for systematic reviews and meta-analyses: the PRISMA statement. Ann Intern Med 2009;151:264-269. doi:10.7326/0003-4819151-4-200908180-00135

24. Higgins JPT, Green S. (Eds.) Cochrane Handbook for Systematic Reviews of Interventions Version 5.1.0. Volume 2014. London, UK: The Cochrane Collaboration; 2011. Available from: www.handbook. cochrane.org

25. Higgins JP, Altman DG, Gotzsche PC, et al; Cochrane Bias Methods Group; Cochrane Statistical Methods Group. The Cochrane Collaboration's tool for assessing risk of bias in randomised trials. BMJ 2011;343:d5928. doi:10.1136/bmj.d5928

26. Arber N, Eagle CJ, Spicak J, et al; PreSAP Trial Investigators. Celecoxib for the prevention of colorectal adenomatous polyps. N Engl J Med 2006;355:885-895. doi:10.1056/NEJMoa061652

27. Lieberman DA, Weiss DG, Bond JH, Ahnen DJ, Garewal H, Chejfec G. Use of colonoscopy to screen asymptomatic adults for colorectal cancer. Veterans Affairs Cooperative Study Group 380. N Engl J Med 2000;343:162-168. doi:10.1056/NEJM200007203430301

28. Schreuders EH, Ruco A, Rabeneck L, et al. Colorectal cancer screening: a global overview of existing programmes. Gut 2015;64:1637-1649. doi:10.1136/gutjnl-2014-309086

29. Wee CC, McCarthy EP, Phillips RS. Factors associated with colon cancer screening: the role of patient factors and physician counseling. Prev Med 2005;41:23-29. doi:10.1016/j.ypmed.2004.11.004

30. Seeff LC, Richards TB, Shapiro JA, et al. How many endoscopies are performed for colorectal cancer screening? Results from CDC's survey of endoscopic capacity. Gastroenterology 2004;127:1670-1677. doi:10.1053/j.gastro.2004.09.051

31. Mutneja HR, Bhurwal A, Arora S, Vohra I, Attar BM. A delay in colonoscopy after positive fecal tests leads to higher incidence of colorectal cancer: A systematic review and meta-analysis. J Gastroenterol Hepatol 2020 Dec 22. doi:10.1111/jgh.15381 